\title{
The importance of structural diversity of spores in the taxonomy of Aspergillus (section Nigri)
}

\author{
M.F. Simões*, C. Santos* and N. Lima* \\ *IBB-Institute for Biotechnology and Bioengineering, Centre of Biological Engineering, Micoteca da \\ Universidade do Minho, Campus de Gualtar, Braga, Portugal
}

Species of Aspergillus from section Nigri, also known as black aspergilli, are distributed worldwide and have been widely used for purposes of various types such as: biotechnological, industrial, medical, among others. They have been extensively studied for being the causing agents of biodeterioration of commodities and food [1].

Within this section, new species have been recently described and among them Aspergillus ibericus [2] and Aspergillus uvarum [3] were both isolated from grapes. The polyphasic approach used in the analysis of these species, either by morphological techniques as well as by molecular methods, allowed not only their characterisation but also their consequent separation from all others in the section. Microbial taxonomy has the identification of species as one of its fundamental goals. Data such as: morphological characteristics description, physiological and biochemical properties, ecological roles, and societal risks or benefits, are key elements in any fungal identification process [4]. But, this process is subjected to periodic changes due to frequent revisions of the taxonomic schemes, therefore becoming time consuming and more demanding and difficult, even for skilled specialists. Furthermore, each taxonomic group has specialised literature, terminology and characters [5]. This takes place since identifications have difficulties of consensual naming, depending on the criteria used and the amount of information available when producing all data. It is increasingly becoming clear that, to better achieve a more accurate concept of species, microbial identification and authentication require a polyphasic approach to produce consistent, useful and quality data [6].

Characterisation of morphologic and structural aspects of spores from Aspergillus strains, section Nigri, has been carried out using scanning electron microscopy (SEM) and intends to contribute to the associated data of the strains from this section.

Colonies from 13 Aspergillus from section Nigri, from the Micoteca da Universidade do Minho (MUM), were grown at $25^{\circ} \mathrm{C}$ for 3 or 4 days in malt extract agar directly mounted in a sterilised SEM stub. The samples were covered with a mixture of gold and platinum (80/20\%) and then examined in the SEM [NanoSEM - FEI Nova ${ }^{\mathrm{TM}}$ 200 (FEG/SEM); EDAX - Pegasus X4M (EDS/EBSD)]. A stereomicroscope (Leica MZ12.5) was used to have a clear image comparison of the size of the conidial head.

Although the analysed strains presented dimensional, morphological and structural diversity, common or typical features could be inferred and related to each taxon like those represented in Fig 1.

From the SEM analysis we were able to conclude that in the section Nigri of genus Aspergillus the spore wall ornamentation, and its size and shape continue to present themselves as important primary diagnostic traits for species differentiation. 


\section{References}

1. Abarca M.L. et al., Antonie van Leeuwenhoek, 86:33-49, 2004.

2. Serra R. et al., Mycologia 98:295-306, 2006.

3. Perrone G. et al., Int. J. Syst. Evol. Microbiol., 58:1032-1039, 2008.

4. Lima N. et al., Proceeding Book of the XXVII ECCO Meeting, Ghent, Belgium, 11-12, 2008.

5. Santos C. et al., Proceeding Book of the XXVII ECCO Meeting, Ghent, Belgium, 65, 2008.

6. Keys C.J. et al., Infect. Genet. Evol., 4:221-242, 2004.

Acknowledgements:

The research leading to these results has received funding from the EMbaRC project (FP7 grant agreement No. FP7-228310). M.F. Simões acknowledges FCT - Portugal for the scholarship SFRH/BD/64260/2009.

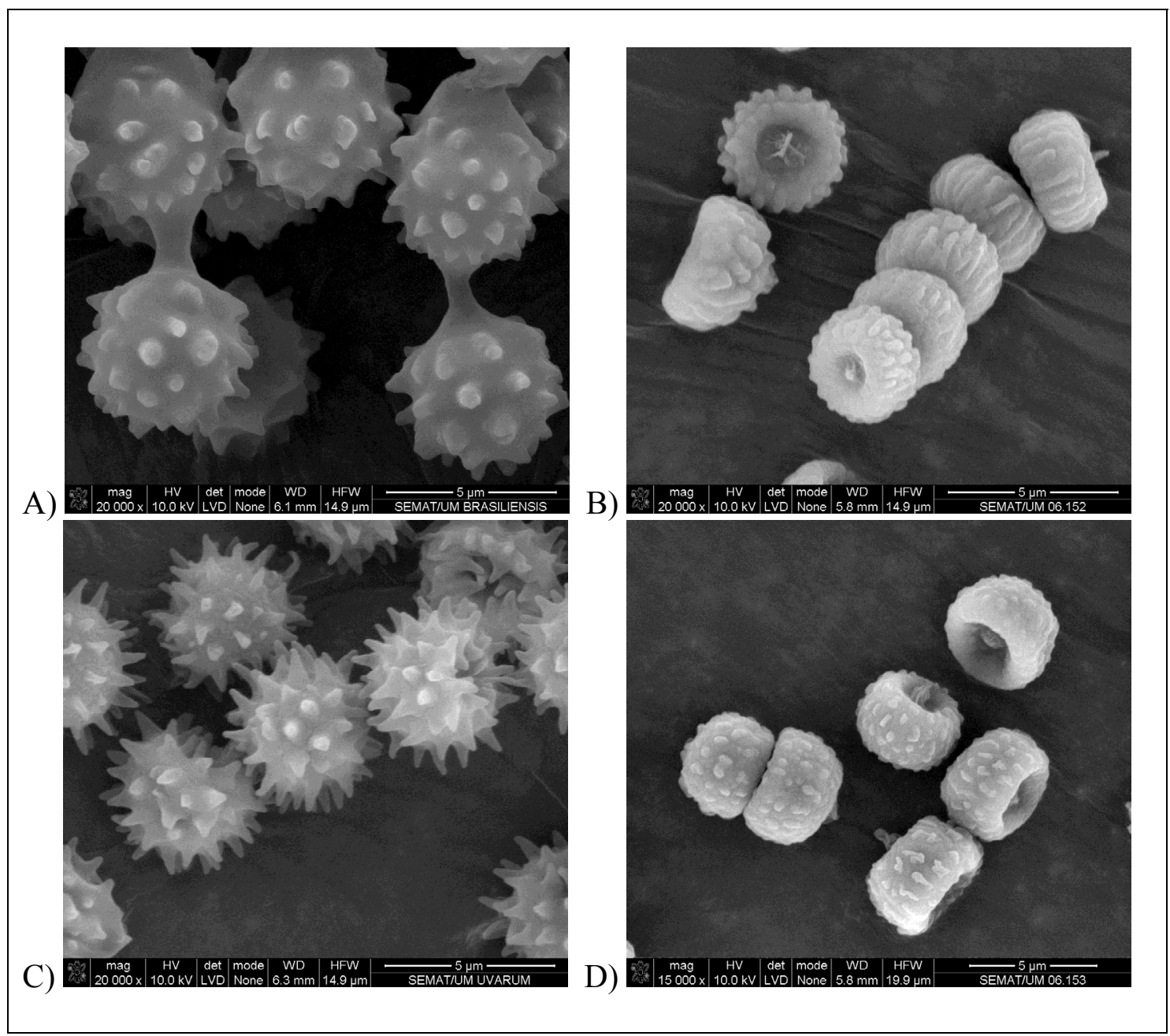

Fig. 1 - Representative Scanning Electron Microscopy images of spores of: A) A. brasiliensis MUM 06.179, B) A. tubingensis MUM 06.152, C) A. uvarum MUM 08.01 and D) A. vadensis MUM 06.153, where spore ornamentation differences, as well as size and shape are clearly seen. Bars: $5 \mu \mathrm{m}$. 